Goal Orientation, Evaluative Fear, and Perceived Coach Behavior among Competitive

Youth Swimmers in Germany and Japan

\author{
Dorothee Alfermann ${ }^{1}$, Guido Geisler ${ }^{2}$ and Yoshinori Okade ${ }^{3}$ \\ ${ }^{1}$ University of Leipzig, Leipzig, Germany \\ ${ }^{2}$ University of Edinburgh, Edinburgh, UK \\ ${ }^{3}$ University of Tsukuba, Ibaraki, Japan
}

Keywords: Collectivism, cross-cultural psychology, individualism, satisfaction

Corresponding author:

Dorothee Alfermann

Faculty of Sport Science

Jahnallee 59

D-04109 Leipzig, Germany

Phone: +493419731633

Fax: +493419731639

E-mail address: alfermann@uni-leipzig.de 


\begin{abstract}
Objectives: The primary objective of this study was to compare youth athletes from two culturally distinct nations (Germany and Japan) on key aspects of their psychological makeup (goal orientation, fear of evaluation) and perceived coach support.

Design: Data were obtained with questionnaires distributed during a training session and analyzed with regression as well as multivariate and univariate analyses of variance.
\end{abstract}

Method: There were 56 German (30 female, 26 male) and 117 Japanese (60 female, 57 male) swimmers with a mean age of $13.2(S D=2.0)$ and $14.1(S D=1.8)$, respectively. All of the athletes participated regularly in high-level competitions.

Results: As hypothesized, the German athletes were more task than ego oriented and more task focused than the Japanese, whereas the latter group had higher ego orientation scores than the German swimmers and put similar emphasis on both task and ego concerns. Contrary to expectations, there were no differences between the national cohorts in evaluative fear. The Japanese swimmers perceived their training climates to be more competition oriented than did the German participants. In terms of coach-athlete interactions, the German athletes perceived significantly more instruction, positive feedback, and social support than the Japanese. Coach variables contributed significantly to the Japanese participants' feelings of satisfaction, with no such correlations in the German sample.

Discussion: Results are interpreted through cultural traditions and may be partly explained by differences in individualism. Practical recommendations for coaches and consultants are offered in light of the research findings.

Key words: Collectivism, cross-cultural psychology, individualism, satisfaction 


\section{Goal Orientation, Evaluative Fear, and Perceived Coach Behavior among Competitive Youth Swimmers in Germany and Japan}

\section{Introduction}

Adolescence is often a critical time in sport. During this period, young people are typically in the developmental stage of their athletic careers (cf. Wylleman \& Lavallee, 2004), but it is also a time in which they are increasingly prone to dropout due to the dual demands of sport and school commitments (cf. Alfermann \& Stambulova, 2007). To balance these demands successfully, youth sport competitors rely on various personal and social resources, and these resources are functions of the individual self as well as the performer's social micro-environment. In contemporary sport psychology, examination of young athletes' resources puts particular emphasis on the "athletic triangle" (the athlete, parents, and coaches), with each component playing a part in how participants respond to the challenges placed before them. However, responses can differ between individuals, even when personal and/or environmental circumstances appear similar. To illustrate, Wylleman and Reints (2010) found that young swimmers who had recently entered an elite sport school were primarily focused on coping with academic requirements, whereas tennis players from the same school reported greater concerns about meeting the demands of a new and more ambitious training schedule. As mentioned, one source of such differences is the nature of athletes' personal resources, and the current literature suggests that adherence and success in sport are largely associated with individual levels of achievement motivation. That is, meeting the heavy demands of training and competition relies considerably on intrinsic motivation (Wylleman \& Reints, 2010), persistence (Bußmann \& Alfermann, 1994), and task orientation (Duda, 1992). Task orientation is concerned with skill mastery and improvement, with relatively little regard for outcome; this is in contrast to an ego-oriented focus on objective performance outcomes and interpersonal comparison (Duda, 1992). 
In terms of social resources, parental support has been shown to have a significant effect on skill development in youth sport competitors (Wylleman \& Reints, 2010) while the other main social resource — and remaining component of the aforementioned athletic triangle - is the support offered by coaches. This is underlined by Alfermann, Lee, and Würth (2005), who found that the progress of adolescent swimmers in the developmental phase of their athletic careers was closely and positively related to their coaches' instructional and feedback behavior.

Research into youth sport, however, has largely been carried out in the West. Fewer studies have addressed important variables for successful sports development in non-Western countries and whether athletes from diverse cultures differ in those variables. Among the small but growing body of research conducted to date, the most notable East-West differences have pointed to goal orientations on the athletes' part (e.g., Geisler \& Leith, 2010; Hayashi \& Weiss, 1994; Isogai, Brewer, Cornelius, Etnier, \& Tokunaga, 2003; Kim \& Gill, 1997) and to motivational climate and coach behaviors in the social micro-environment (e.g., Chelladurai, Imamura, Yamaguchi, Oinuma, \& Miyauchi, 1988; Chelladurai, Malloy, Imamura, \& Yamaguchi, 1987; Yoshida, Matsuo, Yamamoto, \& Taniguchi, 1998). To account for such variation, researchers often invoke the culturally-centered dimensions of individualism (IND) and collectivism (COL), as outlined below.

\section{Individualism and collectivism as important cultural dimensions}

Individualism is a worldview that centralizes the personal (e.g., personal goals, personal uniqueness, and personal control) and puts social conditions at the periphery. Thus, people sample information from the standpoint of self (I think ...). Collectivism, on the other hand, is a social way of being that is oriented toward in-groups (e.g., family, clan, religious groups, etc.) and away from out-groups. People gather information from the standpoint of their group (they think ...), meaning that collectivism prioritizes group solidarity and social relationships (Markus \& Kitayama, 1991). Research suggests that people in many Asian 
countries exhibit a stronger sense of collectivism, interdependence, hierarchy, and community than those in Europe and North America, where the greater focus is on independence, individuality, and horizontal relationships (Kim \& Gill, 1997; Markus \& Kitayama, 1991; Moy, 1992). Individualism and collectivism are thought to have consequences for a variety of psychological traits and states, such as self-concept, well-being, social relations, emotions, cognition, and motivation (Oyserman, Coon, \& Kemmelmeier, 2002). Accordingly, it is reasonable that athletes from Eastern and Western nations might also possess competitionrelated orientations that reflect these culturally-derived attributes, but an interesting pattern emerges when examining Germany and Japan as specific target populations. To explain, differences between these countries have been found on individualism, with Japan scoring lower than Germany, but in contrast to the East-West assumptions noted above, collectivism scores in both nations have been measured as relatively equal (see Oyserman et al., 2002). The question therefore arises as to the applicability of the IND-COL framework in German and Japanese sport, and whether young athletes in the two countries can be separated along these lines on attributes considered relevant for success.

The construct of motivation provides a further inconsistency from an IND-COL perspective. The dictates of individualism suggest that athletes in IND countries should score higher on competitive motivation than those in COL cultures, but in an analysis of the World Value Survey (WVS), Hayward and Kemmelmeier (2007) found no such correlation between IND and competitive values. This may be due to the fact that competition in many presumed COL nations actually begins from an early age. Young people in Japan, for example, must compete for the best places in the educational system (often as early as kindergarten) by maintaining top academic standing and enduring the pressure of entrance exams. Sporting excellence can sometimes provide an alternative method of admission to favored high schools or colleges (Kai, 1994), and as a result, the associated competition may foster a stronger ego 
orientation than in Germany and other more IND Western nations, where access to education is a right.

\section{Cross-cultural comparison of athletes' measures of motivation}

High levels of ego and win orientation have indeed been documented among Japanese participants in both sport and physical education (PE) settings. Research by Isogai et al. (2003) found higher ego and lower task orientation scores among Japanese PE students than among Americans, while one of the first comparative studies with competitive athletes in Japan examined the achievement motivation of Japanese and Anglo-American marathon runners (see Hayashi \& Weiss, 1994). In the latter study, the researchers found that the American runners were significantly higher on competitiveness (the desire to strive for sport achievement) than the Japanese, whereas the Japanese runners scored significantly higher on win orientation (the desire to win in interpersonal competition). To explain their findings, Hayashi and Weiss concluded that American athletes were more likely to describe success as overcoming competitive challenges. The Japanese, on the other hand, put greater emphasis on achieving success through winning.

The above-mentioned findings notwithstanding, there is a further and interesting caveat in East-West patterns of motivation. In a comparison of goal orientations among culturally diverse soccer players, Geisler and Leith (2010) observed that Japanese competitors had higher ego ratings as well as higher combined task/ego scores than Canadian and German players. Thus, the underlying suggestion is that young Japanese athletes employ multiple criteria in their judgments of success, and that the demonstration of superiority (winning) is complemented by a similarly strong focus on task mastery.

Equally noteworthy is the effect of failing in Japan. Failure in Japanese sport and other achievement contexts is often associated with a sense of shame, and the desire to prevent this shame can be satisfied through an ego-oriented focus on winning (Hayashi \& Weiss, 1994). Heine et al. (2001) also found significant differences between Japan and North America in 
people's reactions to failure (on academic tasks). The Japanese treated failure as an impetus to improve one's skills or abilities and increase the level of effort (i.e., a task-oriented approach) while North Americans showed a reduced effort after failure and usually lost interest in the task, but Heine and colleagues' research did not examine such reactions in athletic settings. From a COL or perhaps group-oriented viewpoint, it is possible that in Japan, the shame of failure (or losing) in competitive sport arises from failing to meet one's obligations to teammates, coaches, and supporters. Obligation is seen as a traditional value in Japanese sport (Nagaki, 1998), and the consequences of failure are likely to include unfavorable evaluations from members of the athlete's socially important "miuchi” (or in-group). In other words, losing may have stronger social ramifications than in the West, and the resulting self-presentational concerns should be heightened by a Japanese predisposition toward negative self-evaluation and acknowledgment of personal weaknesses (Kashiwagi, 1986; Kitayama, 2002) as well as a cultural susceptibility to embarrassment (Singelis \& Sharkey, 1995)—phenomena which are also evident in Japanese sport (Kozuma, 2009), and which may subsequently affect athletes' perceptions, cognitions, and behavior.

The importance in Japanese society of meeting the social expectations of one's group (see Markus \& Kitayama, 1991) reflects the significance of social comparison processes. Since ego orientation and evaluative concern are both functions of social evaluation, it is possible that the Japanese scores on these measures would be higher than what is typically found in most Western societies. Nevertheless, cross-cultural differences in evaluative fear have remained largely unaddressed, both in team and individual sport contexts. An exception is the work of Geisler and Leith (2009), which showed that university soccer players in Japan had considerably higher evaluative concern than players in Canada and Germany. Moreover, the Japanese concerns were team-oriented and focused on players' in-groups. These results suggest that the importance of maintaining "face" in Japanese life (Whiting, 1977) can be extended to Japanese sport through a cultural sense of group obligation and the corresponding pressure to 
win, but it is unclear whether such group obligation plays a similar role in individual sports as it presumably does within teams.

\section{Cross-cultural differences in perceived coach behavior}

Other East-West differences have been demonstrated in the areas of leadership, coaching behavior, and motivational climate. Ntoumanis and Biddle (1999) suggest that the meaning of success, failure, and achievement in different cultures is primarily dictated by the prevailing motivational climate - the environment's situational goal structure as created by significant others, and the extent to which it is task (mastery) and/or ego (performance) involving. In an earlier study, Chelladurai et al. (1987) found that Japanese PE students preferred more supportive leadership than Canadian students, and that Japanese participants in modern (Western) sports had a greater preference for participative decision-making than their Canadian counterparts. Chelladurai et al. (1988) then conducted follow-up research with male intercollegiate athletes from the same two countries and looked at their perceptions, preferences, and satisfaction with coaching style. The Japanese athletes perceived their coaches to display higher levels of autocratic behavior while the Canadians perceived higher levels of training and instruction, democratic behavior, and positive feedback (more taskoriented leadership). The Canadians also expressed more satisfaction than the Japanese athletes with both the leadership style of their coaches and their own personal performance outcomes. Chelladurai et al. (1988) summarized the findings by stating that Japanese leadership is characterized by both autocratic and supportive behavior, while in Canada, it is mainly democratic and task oriented.

Additional research on university athletes in Japan, Germany, Canada, USA, China, Russia, and South Africa suggests that Japanese coaches do not closely monitor their relationships with all of the athletes on their teams (Yoshida et al., 1998). This is backed up by Polster's (2004) comparative essay on coaching and sports management in Japan and Germany, which claims that Japanese coaches are granted less social respect than German 
coaches (partly because of inefficient sports management systems) and that traditional Japanese group dynamics inhibit interpersonal coach-athlete cooperation. In light of the available findings, then, there is reason to believe that the "coach" portion of the aforesaid athletic triangle can have differential effects on athletes from diverse cultures, but GermanJapanese comparisons are scarce beyond those of Polster (2004) and Yoshida et al. (1998).

\section{Objectives of the study}

It is important to clarify how selected cultures are defined when contrasting the socialization of athletes from different cultural backgrounds. In this study, "culture" was simply equated with the country in which the sampled athletes competed. It is also important to differentiate between those things that are national culture-specific and those that are standard functions of sport. On the one hand, the values and norms of a given culture create an environment that socializes its members and teaches them how to structure, process, and weigh incoming information. These values and norms can affect views on competition, motivation, and sports leadership, and Chelladurai et al. (1988) call this the "cultural influence" perspective. By the same token, athletes in competitive sports are also confronted with universal demands that are independent of culture, such as striving for success. From that viewpoint, one could expect similar reactions across all cultures to the demands of competitive sport, a notion which Chelladurai et al. (1988) refer to as the "athletic imperatives hypothesis".

Taking the extant research into account, the primary objective of this study was to compare athletes from two culturally distinct nations on their psychological make-up and perceived coach support during the developmental phase of their athletic careers. More specifically, a cross-cultural psychology framework was used to analyze self-reported goal orientation, evaluative concern, perceived motivational climate, and perceptions of coach behavior among adolescent competitive swimmers in Germany and Japan. These variables are regarded as important and influential factors in athletic development; accordingly, the 
research sought to assess the corresponding levels of congruence (athletic imperatives hypothesis) and/or variation (cultural influence hypothesis) between both nations. Germany and Japan served as the relevant target cultures because of their geographical distinctions and the aforementioned national differences on IND (but not necessarily COL) measures. Their selection was also based on the dearth of German-Japanese comparisons in sport. Aside from the Geisler and Leith $(2009,2010)$ research, one of the few such studies was conducted by Teipel et al. (1998), who showed that Japanese soccer players experienced more stress than German participants in various game and training situations. However, each of these investigations involved team sport (soccer) athletes, and there are few (if any) comparisons of individual sport competitors in these nations. Moreover, Geisler and Leith examined players at the intercollegiate (university) level. In Germany, university sport is taken less seriously than in Japan and less seriously than club sport overall, with a lower level of performance and win-oriented motivation than competitive club-based athletes (see Geisler \& Leith, 2010). Consequently, the findings should help fill a current knowledge gap about competitive youth sport in both nations while contributing to existing theory in cross-cultural sport psychology.

An additional purpose of the study stems from the fact that the most recent research into coach behavior and motivational climate in Japan was conducted some decades ago and in team sport contexts. It is therefore unclear whether those results remain valid today, especially for individual sport athletes. In practical terms, the study should also inform best practice for youth-level coaches in the training and development of individual sport competitors in German/European and Japanese/East Asian settings.

On the basis of literature review and the cultural influence perspective, the following hypotheses were proposed:

1. The Japanese swimmers were expected to score higher on ego orientation and lower on task orientation than the German swimmers. 
2. The Japanese swimmers were expected to report a stronger fear of negative evaluation than the German swimmers.

3. The motivational climate within training groups was expected to differ between the countries, with the Japanese athletes reporting a stronger performance climate than the German group and the Germans reporting a stronger mastery climate than the Japanese.

4. Similarly, the Japanese swimmers were expected to perceive their coaches' behavior as more autocratic than in Germany, with less Training and Instruction and less Positive Feedback.

5. However, based on the findings of Chelladurai et al. (1988), the Japanese swimmers were also expected to perceive their coaches as more socially supportive and closer to the athletes.

\section{Method}

\section{Participants}

The participants were male and female swimmers from 12 to 18 years of age. There were 56 German (30 female, 26 male) and 117 Japanese (60 female, 57 male) swimmers, with a mean age of $13.2(S D=2.0)$ and $14.1(S D=1.8)$, respectively. Participants were members of training groups with one head coach, and in the case of coach variables, the athletes were instructed to rate that coach. Apart from 33 Japanese participants in all-female training groups, the swimmers practiced in combined-gender sessions. The average group size in Japan consisted of 14.4 females and 12.6 males. In Germany, it was 6.8 males and 9.0 females, which resulted in a significant difference in group size between the two nations, $F(1$, $122)=39.4, p<.001, \eta^{2}=0.24$.All participants took part in several weekly training sessions, but the German swimmers practiced more hours per week than the Japanese $\left(M_{\mathrm{G}}=16.1, S D_{\mathrm{G}}\right.$ $\left.=4.1 ; M_{\mathrm{J}}=11.2, S D_{\mathrm{J}}=4.3 ; F(1,135)=34.47, p<.001, \eta^{2}=0.20\right)$. Lastly, the training groups in both countries were highly performance oriented. More than $90 \%$ of the swimmers 
participated regularly (at least once or twice per month) in competitions, and $70 \%$ competed at the national or international level.

\section{Procedure}

After the coaches had given consent to the study, the swimmers were contacted in their training groups and asked if they would volunteer to participate. Parents of underage swimmers were asked for their permission, and all participants were assured of anonymity and confidentiality in the recording, analysis, and presentation of data. The research questionnaires were distributed at a regularly scheduled training session. In Germany, the swimmers then completed the questionnaires at home and returned them on the next training day. In Japan, the questionnaires were completed on site and collected the same day, and the difference in the two data collection methods reflected the coaches' wishes in each country. Interestingly, this procedural discrepancy may underline a cultural difference in itself. Given the previous references to obligation in Japan, it is conceivable that the Japanese coaches, having agreed to take part in the research, considered it their duty to ensure a prompt return of the completed questionnaires.

\section{Measures}

Task and ego orientation were measured with the German (Rethorst \& Wehrmann, 1998) and Japanese versions (Isogai et al., 2003) of the Task and Ego Orientation in Sport Questionnaire (TEOSQ; Duda, 1992). All scales had satisfactory internal consistencies between $\alpha=.75$ and .78 in both languages.

Fear of negative evaluation was assessed with the brief version of the Fear of Negative Evaluation scale (FNE; Leary, 1983). The German items were developed by Vormbrock and Neuser (1983) while the Japanese version was developed by Ishikawa, Sasaki, and Fukui (1992). Four items in the Japanese version had low or even negative item-total correlations, but quite high correlations in the German version: item 3 (I am frequently afraid of other people noticing my shortcomings; -.018/.502), item 4 (I rarely worry about what kind of 
impression I am making on someone; .002/.772), item 9 (I am usually worried about what kind of impression I make; -.219/.709), and item 11 (Sometimes I think I am too concerned with what other people think of me; -.365/.795).It is unlikely that these items were culturally charged as they were not noticeably different in tone or language from the rest of the questionnaire, but because of their low reliability, the FNE scale was reduced to eight items. This scale had high internal consistency of $\alpha=.93$ (German version) and .88 (Japanese version) in our samples.

Perceived coach behavior included leadership, motivational climate, and closeness. Perceived leadership behavior was measured by the German and Japanese versions of the Leadership Scale for Sport (LSS), originally developed by Chelladurai and Saleh (1980). The German version was developed in the first author's lab (Alfermann, Saborowski, \& Würth, 1997) and has been tested extensively with young athletes in different sports and of varying skill levels (e.g., Alfermann, Lee, \& Würth, 2005). The alpha values for the five subscales in this study were acceptable and ranged between .80 (Training and Instruction - TI), .76 (Positive Feedback - PF), .77 (Social Support - SO), .68 (Autocratic Behavior - AB) and .63 (Democratic Behavior - DB). The Japanese version was developed by Chelladurai, Imamura, and Yamaguchi (1985). The alpha values for the subscales in this study ranged between .74 (TI), 72 (SO), .69 (PF), and .63 (DB). Unfortunately, the Japanese version of the AB scale did not reach an acceptable level of internal consistency $(\alpha=.20)$. This problem of low reliability on the $\mathrm{AB}$ scale has also been documented in other studies (cf. Zhang, Jensen \&Mann, 1997), and as a result, the variable was omitted in the data analysis. Thus, the remaining four scales of the LSS were used to measure perceived instructional behavior of the coaches (TI), positive and encouraging feedback given by the coaches to the athletes (PF), social support for group members and for developing team cohesion (SO), and consideration of athletes' suggestions and wishes in the training process (DB). 
In addition to leadership behavior, motivational climate within the training group was assessed via the original Perceived Motivational Climate in Sport Questionnaire (PMCSQ; Walling, Duda, \& Chi, 1993). The German version of the PMCSQ (Alfermann et al., 1997) contains 15 items that measure performance climate ( 9 items) and mastery climate (6 items). The Japanese version was translated and pilot tested in various sport contexts at the third author's institution. Alpha values of the scales were satisfactory to very good, with $\alpha=.84$ for both the German and Japanese scales for performance climate and $\alpha=.61$ (German scale) and .78 (Japanese scale) for mastery climate.

The Closeness scale was translated from Jowett and Ntoumanis' (2004) original British version and consisted of five items measuring the closeness of the coach-athlete relationship as perceived by the athletes (e.g., "I can trust my coach"). The German and the Japanese versions both had high internal consistency ( $\alpha=.84$ and .87 , respectively).

Finally, athletes were asked for their satisfaction with the coach and the training that they received. These data were assessed on two rating scales, ranging from 1 (not at all satisfied) to 7 (very satisfied). Scores on the scales were then averaged.

\section{Statistical Analyses}

Differences between male and female athletes on the dependent variables were addressed with multivariate and univariate analyses of variance. No gender differences emerged. Therefore, the data of male and female athletes were combined in all further analyses. Cross-cultural differences between the German and Japanese athletes were then tested separately with multivariate analyses of variance (MANOVA). This was followed by corresponding univariate analyses of variance (ANOVA). Additional analyses examined differences in the correlational pattern of perceived coach behaviors and correlations between coach behavior and athletes' satisfaction with both their coaches and their training. This was done in consideration of previous research with the LSS, which showed correlations between 
subscales (Cumming, Smith, \& Smoll, 2006). Due to some missing values, the number of participants varied in the analyses.

\section{Results}

All descriptive statistics and the results of ANOVAs can be found in Table 1. Partial $\eta^{2}$-values were included in addition to the usual $p$-values. The partial $\eta^{2}$ is the proportion of total variability attributable to a factor and can thus be regarded as an estimate of effect size. When interpreting the $\eta^{2}$ values, we rely on Cohen's suggestions (1988) that $\eta^{2}=0.01$ represents a small effect size, 0.06 a medium effect size, and 0.14 a large effect size.

As per the first hypothesis, the German swimmers scored significantly higher on task orientation and lower on ego orientation than the Japanese swimmers. There was a moderate to large difference in ego orientation $\left(\eta^{2}=.12\right)$. The finding for task orientation was also significant, but the difference was relatively small $\left(\eta^{2}=.02\right)$. Contrary to the second hypothesis, however, there were no significant differences in fear of negative evaluation.

In terms of motivational climate and the third hypothesis, there were no significant differences in mastery climate between the two countries but the Japanese swimmers reported a significantly more performance-oriented environment than the German athletes. Here, too, the difference between the two cohorts was moderate to large $\left(\eta^{2}=.11\right)$.

Regarding the leadership behavior of coaches, there was only partial confirmation of the fourth and fifth hypotheses. That is, the German athletes perceived significantly more instructional behavior $\left(\eta^{2}=.19\right)$, more positive feedback $\left(\eta^{2}=.10\right)$, and more social support $\left(\eta^{2}=.13\right)$ than the Japanese athletes. The associated $\eta^{2}$ values indicate that these differences were large or moderate to large. The German swimmers also reported a higher degree of closeness with their coaches than the Japanese, but the difference was less pronounced $\left(\eta^{2}=\right.$ $.03)$.

Correlations among the coach variables were examined in order to more closely address cross-cultural differences in the athletes' perceptions of their coaches (as suggested 
by the previous review of literature). Results showed that both the Japanese and German athletes perceived high correlations between the coach variables. However, there was one exception. As can be seen from Table 2, the relationship of coach behavior variables with performance climate was the main difference between the Japanese and German swimmers. Whereas the German athletes perceived their coaches' behavior as negatively related to a performance climate, the Japanese participants saw such a relationship as positive. For them, all coach behaviors, including those associated with performance climate, were rated positively.

The final analysis addressed relationships between perceived coach behavior and the athletes' satisfaction with the coach and with their training. The Japanese results showed a positive correlation between satisfaction and perceived coach behavior while no such pattern emerged in the German sample (Table 2). In order to further examine possible differences, separate regression analyses were conducted for the participants of the two nations, with satisfaction as the criterion variable and the coach variables as predictors. Overall, satisfaction was not significantly different between the two nations, as indicated by the last line in Table 1. For the Japanese swimmers, though, coach variables contributed significantly to satisfaction $\left(R^{2}=0.400 ; F(7,94)=8.28, p<.001\right)$, with positive feedback $(\beta=0.362)$ and closeness $(\beta=0.425)$ contributing the most to this relationship. For the German swimmers, $R^{2}$ was only $0.065(F(7,32)=0.247, p=.97)$, which means that none of the coach variables were related to the German athletes' level of satisfaction.

\section{Discussion}

This study found differences between German and Japanese competitive youth swimmers in their goal orientations, the perceived motivational climates of their training environments, and their perceptions of coach behavior as well as in factors associated with that behavior. Consequently, there is reasonable evidence to suggest that athletic development 
occurs under different sets of conditions for the two national cohorts and that these differences are associated with both personal and coach/environmental variables.

\section{Personal variables}

The first hypothesis predicted that the Japanese swimmers would have higher ego orientation scores than the German swimmers, whereas the Germans were expected to score higher on task orientation than the Japanese. The hypothesis was confirmed, which is consistent with the literature showing a strong ego emphasis (or focus on winning) among sport participants in East Asia (e.g., Hayashi \& Weiss, 1994; Isogai et al., 2003; Kim \& Gill, 1997; Kim, Williams, \& Gill, 2003).

The notion that winning is a highly valued and celebrated feature of Japanese sport may have its origins in the 1935 establishment of Japan's National Association of (Amateur) Sport, the sporting creed of which includes the winning principle or the precept that players are honor-bound to strive to win (Kusaka, 2006, p. 30). Further historical evidence of the Japanese pressure to win is provided by Pempel (1998), whose essay on contemporary Japanese sport and the country's national and international identity refers to a judo competitor who suffered no embarrassing defeats during a string of 203 consecutive bouts, but who retired at his peak because of feelings that he could no longer maintain this form. Also mentioned is the well-known story of the Japanese marathoner who later committed suicide after earning only bronze at the 1964 Tokyo Olympics and letting his country down (Pempel, 1998). With this historical perspective as a backdrop, there is reason to believe that the tendency toward higher ego orientation among the current study's Japanese swimmers was fostered by the aforesaid feelings of obligation (Nagaki, 1998) and desire to prevent lossrelated shame (Hayashi \& Weiss, 1994) and embarrassment (Singelis \& Sharkey, 1995). It is equally likely that this tendency is a product of a culturally-specific athlete-coach relationship whereby socialization into sport includes the traditional cycle of coaches instilling ego- or 
win-oriented thinking in their athletes - just as their own coaches did with them (see Kozuma, 2009).

Continuing with the first hypothesis, the difference in task orientation also reached significance as predicted, with higher task scores among the German swimmers. However, this difference was relatively small, and the fact that scores were in the high range for both groups supports the existing theory that East Asian competitors are not only ego-focused, but that they tend to embrace both task and ego goal orientations to a similar extent. While this pattern reflects multiple criteria that Japanese athletes use to define success and enjoyment in sport, it may also serve an important adaptive function. To illustrate, Smith, Balaguer, and Duda (2006) showed that a combination of high ego orientation with a moderate or high task focus can prevent the maladaptive motivational patterns often associated with ego-oriented athletes. Thus, when the current results are combined with previous research findings, it appears that athletic development in Japan entails a similarly strong emphasis on task mastery and performance quality as it does on winning and being the best performer or team. From an IND/COL standpoint, it also suggests that the lower level of IND in Japan and other East Asian countries (as per Oyserman et al., 2002) does not necessarily inhibit task-oriented goal attainment in sport.

The expectation that the Japanese participants would report higher evaluative concern was the only hypothesis that could not be confirmed. At first glance, the lack of significant differences between the two groups might be explained via the "athletic imperatives" view (Chelladurai et al., 1988) that universally endorsed performance requirements in sport lead to a degree of cultural congruence in thoughts about competition-and that this congruence includes evaluative fear or self-presentational concern. However, the current finding contradicts Geisler and Leith's (2009) research, which showed markedly higher evaluative fear among Japanese intercollegiate soccer players than among Canadian and German players. To account for the discrepancy between the two studies, it is probable that the Japanese 
swimmers' overall satisfaction with their coaches, training environment, and motivational climate precluded the type of evaluative fear that might be expected in other sports contexts, especially those with longstanding Japanese traditions and codes of behavior (such as kendo, aikido, or baseball). Two further explanations might also warrant consideration. One, the body-revealing nature of swimwear may very well foster universal physical self-presentation cognitions that are common to all swimmers. The second and somewhat opposite possibility is that athletes who choose to swim competitively are sufficiently comfortable with the associated physical self-presentation that they do not experience meaningful concerns about physical evaluation from others. In fact, Prapavessis, Grove, and Eklund (2004) suggest that some people may actually select and participate in sports that emphasize their physique because they are confident about making favorable physical impressions. In either case, differences in evaluative concern between athletes of diverse backgrounds would most likely be negligible. Nevertheless, the discrepancy between the current results and Geisler and Leith's (2009) findings with soccer players underlines the need for further research on selfpresentational thinking among culturally diverse athletes, and individual sport athletes in particular, as well as the possible effects on performance and performance-related cognitions.

\section{Coach and environmental variables}

With respect to motivational climate, the Japanese swimmers perceived their training environments to be more performance oriented than the German participants. This lends support once more to the notion that socialization of young Japanese athletes into competitive sport includes the development of ego- or win-oriented mindsets.

The results for perceived leadership partly corroborated our hypotheses. As expected, the athletes in Germany felt that their coaches provided more positive feedback and more training and instruction than those in Japan. Since IND emphasizes the attention given to individuals, and since the scales used in this research both focused on such individual attention, the differences in perceived coach behaviors can be interpreted as the result of 
cultural differences between Germany and Japan on the IND dimension. Contrary to expectations, however, the Japanese swimmers did not get more social support from their coaches than the German swimmers. In fact, the opposite was true. Given that social support is defined as an interest on the part of coaches in their athletes' individual progress outside of sport (particularly at school), the lesser support from the Japanese coaches may have been an additional manifestation of a lower level of IND in Japan. This contradicts a number of other research findings, as mentioned previously, which showed a greater emphasis on social support among Japanese leaders. However, those studies emphasized a Japanese preference for autocratic style as well, and unfortunately, the low reliability of the Autocratic Style scale meant that this hypothesis could not be adequately tested. As a result, the current findings for social support reinforce Yoshida et al.'s (1998) belief that Japanese coaches do not pay such close attention to individual relationships with their athletes, whether in team or individual sport contexts. They also support Polster's (2004) claim that strong coach-athlete interaction is not a characteristic feature of the Japanese sport experience.

Overall, leadership behavior proved to be a significant variable in differentiating the participants of the two countries, and the differences in perceived coaching behaviors did not fully corroborate those that Chelladurai et al. (1988) found with male intercollegiate athletes in Canada and Japan. These differences, in combination with the fact that Chelladurai and colleagues' research was carried out with university athletes nearly 25 years ago, reinforces the need for further study into perceived and preferred coaching behaviors of youth sport competitors in Eastern and Western nations. It also remains unclear whether the different leadership styles of German and Japanese coaches have differential effects on athletes' skill development or performance. As mentioned earlier, Alfermann et al. (2005) found that Positive Feedback as well as Training and Instruction both affected young participants' progress, but they were unable to collect further follow-up data. Accordingly, it is difficult to 
predict if and/or how this diversity in leadership behavior might differentially affect the competitive performances of the German and Japanese swimmers here.

\section{Limitations and follow-up study}

The study's sampling methodology and data distribution offer additional points to consider. More specifically, there were 56 participants in the German sample and 117 participants in Japan. Given that the cross-national comparison of FNE scores produced a $p$ value of approximately .10, it follows that significant differences might have emerged if a larger number of German athletes had been examined. Similarly, the absence of significant differences between the two cohorts of swimmers may be a function of outliers that occurred in the data. Those outliers were related to the female participants in Germany, who had higher FNE scores than the German males. No such gender differences were seen in Japan, which means that the German females' scores were similar to the scores for both the Japanese females and the Japanese males.

The latter point has implications beyond the research methodology. The construct of social physique anxiety (SPA; Hart, Leary, \& Rejeski, 1989) involves the belief or fear that others are likely to judge one's physique negatively (e.g., as overweight, uncoordinated, or unfit), and as noted, it is possible that the attire of competitive swimmers engenders physical self-presentational thinking (in the form of SPA) that is less evident in other sports. For instance, research by Van Raalte, Cunningham, Cornelius, and Brewer (2004) found female athletes to report significantly greater SPA than male athletes. Thus, if SPA was a factor in the current investigation, the possibility exists that FNE scores of the German females masked differences that actually existed between the German and Japanese males.

To better understand the evaluative and self-presentational concerns of elite and culturally diverse young swimmers, future research should pay greater attention to the details of athletes' evaluative fears. Bray, Martin, and Widmeyer (2000, p. 359) note that it is important to identify, acknowledge, and address the specific concerns of athletes with respect 
to social evaluation. Moreover, Geisler and Leith (2009) showed that the nature of those concerns varies in different cultures. Their research found that Canadian and German soccer players were cognizant of the impressions left on a variety of different people whereas Japanese players' concerns were largely team-oriented and directed toward peers or members of their in-groups. Follow-up study should therefore take such culturally-derived differences into consideration.

Future research might also choose to utilize Elliot and colleagues' 2 × 2 framework of ego orientation (Elliot \& McGregor, 2001) when examining the goal orientations of Japanese competitive athletes. The 2 x 2 model has only recently been incorporated into sport studies, but it is a useful approach because it is comprised of four goal motives - mastery-avoidance, mastery-approach, performance-approach, and performance-avoidance goals. Elliot and Church (1997) believe that mastery-approach goals and performance-approach goals are endorsed by individuals with high perceived competence, and that they generally lead to both adaptive achievement striving and positive affective responses (as task goals are believed to do) because they are based on a desire to approach challenges. Performance-avoidance goals, on the other hand, are associated with low competence perceptions and are rooted in the fear of failure. As such, they are predicted to have a negative influence on affect and to promote maladaptive forms of achievement striving. With a view to the Japanese swimmers in the current study, it is plausible that the presumed pressure to win and the athletes' ego-oriented thinking could ultimately have a positive effect on athletic development and achievement if there is a concomitant presence of performance-approach motives.

A final word is necessary on the IND/COL framework and its role in sport research. It is interesting that results for the first hypotheses mirrored previous findings on similar measures with IND (German) and COL (Chinese and Pakistani) comparison groups. That is, Asghar, Wang, Linde, and Alfermann (2012) found that ratings of task and ego orientation among German soccer and field hockey players followed the same patterns as those of the 
present study's German swimmers. Similarly, the higher ego scores of Chinese and Pakistani players in the Asghar et al. research reflected the results for the Japanese swimmers here. Nevertheless, it has yet to be established that Western and Asian or East Asian athletes indeed adopt IND and COL ways of thinking, respectively, when engaged in training or competition. In today's global environment, the concept of a homogeneous population is no longer realistic, and not all members of so-called IND nations are individualists nor does everyone within a COL society embrace collectivistic customs (Singelis \& Brown, 1995). For that reason, it would be easier to draw conclusions from future cross-cultural research if IND and COL were examined more directly as variables. Possible inventories include the Individualism-Collectivism Scale (ICS; Triandis, 1995) or a sport-specific version of the Twenty Statements Test (TST; Kuhn \& McPartland, 1954). Some investigators have also designed their own questionnaires for specific research purposes, such as Lee and Choi's (2005) scale measuring Triandis' $(1995,2001)$ horizontal and vertical dimensions of IND/COL. Regardless of the instruments used, Kim and Gill (1997) contend that measuring such culturally-derived variables can provide information about various factors in sport, including those that influence task or ego orientation and other indices of motivation.

\section{Practical recommendations}

In closing, this research gives rise to some practical and culture-specific coaching recommendations for the development of training and motivational climates that reflect the social and motivational orientations of athletes (i.e., participants' overall satisfaction).In Germany, task orientation was preferred over ego orientation and mastery climates were rated more positively than performance climates. In fact, the German athletes perceived their coaches' behavior as negatively related to win- or performance-oriented climates and felt that considerable weight was placed on training and instruction, positive feedback, and social support. At the same time, the personal coach variables in this study did not show any significant correlations with the German athletes' overall satisfaction. Accordingly, there is no 
current evidence for increasing the competitive motivation of young German swimmers, nor is there an apparent need to enhance coach-athlete closeness or support beyond current practices. Coaches are therefore advised to cultivate task-focused thinking and masteryoriented environments, with primary emphasis on the swimmers' physical and skill development.

Those responsible for the development of Japanese swimmers, on the other hand, are advised to put equal emphasis on both task (mastery) and ego (competing to win) considerations. This is based on the Japanese participants' dual endorsement of task and ego motives, a finding consistent with the extant findings for East Asian athletes. It also draws from the fact that while the Japanese athletes perceived their coaches' behavior as positively related to a performance climate, all coach behaviors were rated positively. Furthermore, although the Japanese swimmers actually preferred more socially supportive coaching, the results suggest that it is not necessarily imperative to develop strong individual coach-athlete relationships since the swimmers were sufficiently satisfied with their coaches' training methods and the corresponding motivational climate. Thus, there is little reason to conclude that competitive motivation should be de-emphasized in Japan, but coaches of Japanese youth sport might wish to engender performance-approach motives so as to optimize their athletes' adaptation abilities.

There is more to be learned about psychological factors that influence the development of culturally diverse youth athletes. To provide adequate training and support, and to ensure young athletes' continued satisfaction, further consideration must be given to cultural traditions in sport and the role of IND or COL thinking. The practical links between these phenomena and youth sport progress have yet to be fully understood. 


\section{References}

Alfermann, D., \& Stambulova, N. (2007). Career transitions and career termination. In G. Tenenbaum \& R. C. Eklund (Eds.), Handbook of sport psychology (3rd ed., pp. 712733). New York: Wiley.

Alfermann, D., Lee, M. J., \& Würth, S. (2005). Perceived leadership behavior and motivational climate as antecedents of adolescent athletes' skill development. Athletic Insight - The Online Journal of Sport Psychology, 7(2). Retrieved from http://www.athleticinsight.com/Vol7Iss2/LeadershipandMotivation/html.

Alfermann, D., Saborowski, C., \& Würth, S. (1997). Soziale Einflüsse auf die Karriereübergänge bei jugendlichen Athletinnen und Athleten in Großbritannien und den neuen Bundesländern. Entwicklung und Überprüfung der deutschsprachigen Messinstrumente im Projekt. [Social influences on career transitions of adolescent athletes in Great Britain and in the German new federal states. Development and validation of the German scales in the project.] Unpublished Research Report, University of Leipzig: Faculty of Sport Science.

Asghar, E., Wang, X., Linde, K., \& Alfermann, D. (2012). Comparisons between Asian and German Male Adolescent Athletes on Goal Orientation, Physical Self-Concept, and Competitive Anxiety. International Journal of Sport and Exercise Psychology. Manuscript accepted for publication.

Bray, S. R., Martin, K. A., \& Widmeyer, W. N. (2000). The relationship between evaluative concerns and sport competition state anxiety among youth skiers. Journal of Sports Sciences, 18, 353-361. doi: 10.1080/026404100402412.

Bußmann, G., \& Alfermann, D. (1994). Drop-out and the female athlete. A study with track and field athletes. In D. Hackfort (Ed.), Psycho-social issues and interventions in elite sports (pp. 89-129). Frankfurt: Lang. 
Chelladurai, P., Imamura, H., \& Yamaguchi, Y. (1985). Subscale structure of the Leadership Scale for Sports in the Japanese context: A preliminary report. Paper presented at the World University Games Conference, Kobe, Japan.

Chelladurai, P., Imamura, H., Yamaguchi, Y., Oinuma, Y., \& Miyauchi, T. (1988). Sport leadership in a cross-national setting: The case of Japanese and Canadian university athletes. Journal of Sport \& Exercise Psychology, 10, 374-389.

Chelladurai, P., Malloy, D., Imamura, H.,\& Yamaguchi, Y. (1987). A cross-cultural study of preferred leadership in sports. Canadian Journal of Sport Sciences, 12,106-110.

Chelladurai, P., \& Saleh, S. D. (1980). Dimensions of leader behavior in sports: Development of a leadership scale. Journal of Sport Psychology, 2, 34-45.

Cohen, J. (1988). Statistical power analysis for the behavioral sciences. Hillsdale, NJ: Erlbaum.

Cumming, S. P., Smith, R. E., \& Smoll, F. L. (2006). Athlete-perceived coaching behaviors: Relating two measurement traditions. Journal of Sport \& Exercise Psychology, 28, 205-213.

Duda, J. (1992). Motivation in sport settings: A goal perspective approach. In G. C. Roberts (Ed.), Motivation in sport and exercise (pp. 57-91). Champaign, IL: Human Kinetics.

Elliot, A., \& Church, M. (1997). A hierarchical model of approach and avoidance achievement motivation. Journal of Personality and Social Psychology, 72, 218-232.

Elliot, A., \& McGregor, H. (2001). A 2 x 2 achievement goal framework. Journal of Personality and Social Psychology, 80, 501-519. doi: 10.1O37//OO22-3514.80.3.501.

Geisler, G., \& Leith, L. M. (2009, June). A cross-cultural study of self-presentational concern among intercollegiate soccer players (Abstract). 14 ${ }^{\text {th }}$ Annual Congress of the European College of Sport Science (ECSS). Oslo, Norway.

Geisler, G., \& Leith, L. M. (2010, June). An East-West perspective on goal orientation, selfand collective efficacy, and the competition environment in university soccer 
(Abstract). 15 $5^{\text {th }}$ Annual Congress of the European College of Sport Science (ECSS). Antalya, Turkey.

Hart, E. A., Leary, M. R., \& Rejeski, W. J. (1989). The measurement of social physique anxiety. Journal of Sport \& Exercise Psychology, 11, 94-104.

Hayashi, C. T., \& Weiss, M. R. (1994). A cross-cultural analysis of achievement motivation among Anglo-American and Japanese marathon runners. International Journal of Sport Psychology, 25, 187-202.

Hayward, R. D., \& Kemmelmeier, M. (2007). How competition is viewed across cultures: A test of four theories. Cross-Cultural Research, 41, 364-395. doi: $10.1177 / 1069397107306529$.

Heine, S. J., Kitayama, S., Lehman, D. R., Takata, T., Ide, E., Cheung, C., \& Matsumoto, H. (2001). Divergent consequences of success and failure in Japan and North America: An investigation of self-improving motivations and malleable selves. Journal of Personality and Social Psychology, 81, 599-615. doi: 10.1037//0022-3514.81.4.599

Ishikawa, R., Sasaki, K., \& Fukui, I. (1992). Standardization of Japanese version of FNE and SADS. Koudou Ryouhou Kenkyu (Japanese Journal of Behavior Therapy), 18, 10-17.

Isogai, H., Brewer, B. W., Cornelius, A. E., Etnier, J., \& Tokunaga, M. (2003). A crosscultural analysis of goal orientation in American and Japanese physical education students. International Journal of Sport Psychology, 34, 80-93.

Jowett, S., \& Ntoumanis, N. (2004). The Coach-Athlete Relationship Questionnaire (CARTQ): Development and initial validation. Scandinavian Journal of Medicine and Science in Sports, 14, 245-256. doi: 10.1111/j.1600-0838.2003.00338.x.

Kai, T. (1994). A sociological approach to high school athletic club in "Gakureki" society: Towards the possibility for sport as an educational "strategy." Japan Journal of Physical Education, Health and Sport Sciences, 39, 255-266. 
Kashiwagi, K. (1986). Personality development of adolescents. In H. W. Stevenson \& H. Azuma (Eds.), Child development and education in Japan (pp. 167-185). New York: W. H. Freeman.

Kim, B. J., \& Gill, D. (1997). A cross-cultural extension of goal perspective theory to Korean youth sport. Journal of Sport \& Exercise Psychology, 19, 142-155.

Kim, B. J., Williams, L., \& Gill, D. L. (2003). A cross-cultural study of achievement orientation and intrinsic motivation in young USA and Korean athletes. International Journal of Sport Psychology, 34, 168-184.

Kitayama, S. (2002). Cultural psychology of the self: A renewed look at independenc eand interdependence. In C. Hofsten \& L. Backman (Eds.), Psychology at the turn of the millennium, Vol. 2 (pp. 305-322). Florence, KY: Taylor \& Francis.

Kozuma, Y. (2009, Fall). Mental training in motor sports: Psychological consulting for racecar drivers in Japan. Athletic Insight: The Online Journal of Sport Psychology,11(3). Retrieved April 19, 2011, from http://www.athleticinsight.com/Vol11Iss3/Motor.html.

Kuhn, M. H., \& McPartland, T. S. (1954). An empirical investigation of self-attitudes. American Sociological Review, 19, 68-76.

Kusaka, Y. (2006). The emergence and development of Japanese school sport. In J. Maguire \& M. Nakayama (Eds.), Japan, sport, and society: Tradition and change in a globalizing world (pp. 19-34). London: Routledge.

Leary, M. R. (1983). A brief version of the Fear of Negative Evaluation Scale. Personality and Social Psychology Bulletin, 9, 371-376. doi: 10.1177//0146167283093007.

Lee, W. N., \& Choi, S. M. (2005, February). The role of horizontal and vertical individualism and collectivism in online consumers' response toward persuasive communication on the web. Journal of Computer-Mediated Communication, 11(1). Retrieved June 4, 
2011, from http://jcmc.indiana.edu/vol11/issue1/ wnlee.html. doi: 10.1111/j.10836101.2006.tb00315.

Markus, H. R., \& Kitayama, S. (1991). Culture and the self: Implications for cognition, emotion and motivation. Psychological Review, 98, 224-253.

Moy, S. (1992). A culturally sensitive, psychoeducational model for understanding and treating Asian-American clients. Journal of Psychology and Christianity, 11, 358-367.

Nagaki, K. (1998). A comparison of Australian and Japanese judo practitioners' values and attitudes toward sport. In K. Hardman \& J. Standeven (Eds.), Cultural diversity and congruence in physical education and sport (Proceedings of the $10^{\text {th }}$ ISCPES Biennial Conference 1996 in Hachi-ohji, Japan). Aachen: Meyer \& Meyer.

Ntoumanis, N., \& Biddle, S. J. H. (1999). A review of motivational climate in physical activity. Journal of Sports Sciences, 17, 643-665. doi: 10.1080/026404199365678.

Oyserman D., Coon, H. M., \& Kemmelmeier, M. (2002). Rethinking individualism and collectivism: Evaluation of theoretical assumptions and meta-analyses. Psychological Bulletin, 128, 3-72. doi: 10.1037/0033-2909.128.1.3.

Pempel, T. J. (1998). Contemporary Japanese athletics: Window on the cultural roots of nationalism/internationalism. In S. Linhart \& S. Frühstück (Eds.), The culture of Japan as seen through its leisure (pp. 113-137). Albany, NY: SUNY Press.

Polster, H. (2004, October 1). Sports management and the coaching of sports in Germany and Japan-personal experiences. Retrieved May 5, 2011, from http://hdl.handle.net/10086/14198.

Prapavessis, H., Grove, J. R., \& Eklund, R. C. (2004). Self-presentational issues in competition and sport. Journal of Applied Sport Psychology, 16, 19-40.

Rethorst, S. \& Wehrmann, R. (1998). Der TEOSQ-D zur Messung der Zielorientierungen im Sport. [The TEOSQ-D as a measure of goal orientations in sport]. In D. Teipel, R. 
Kemper \& D. Heinemann (Eds.), Sportpsychologische Diagnostik, Prognostik, Intervention (pp. 57-63). Köln: bps.

Singelis, T. M., \& Brown, W. J. (1995). Culture, self, and collectivist communication:

Linking culture to individual behavior. Human Communication Research, 21 ,354389. doi: 10.1111/j.1468-2958.1995.tb00351.x.

Singelis, T. M., \& Sharkey, W. F. (1995). Culture, self-construal, and embarrassability. Journal of Cross-Cultural Psychology, 26, 622-644. doi: 10.1177/002202219502600607.

Smith, A. L., Balaguer, I., \& Duda, J. (2006). Goal orientation profile differences onperceived motivational climate, perceived peer relationships, and motivation-related responses of youth athletes. Journal of Sports Sciences, 24, 1315-1327. doi:

$10.1080 / 02640410500520427$.

Teipel, D., Ichimura, S., Matsumoto, M., Sugiyama, Y., Uemukai, K., \& Kondo, A. (1998). Assessment of psychological stress conditions amongst German and Japanese soccer players. In K. Hardman \& J. Standeven (Eds.), Cultural diversity and congruence in physical education and sport (Proceedings of the 10th ISCPES Biennial Conference 1996 in Hachi-ohji, Japan). Aachen: Meyer \& Meyer Sport.

Triandis, H. C. (1995). Individualism and collectivism. Boulder, CO: Westview.

Triandis, H. C. (2001). Individualism-collectivism and personality. Journal of Personality, 69, 907-924. doi: 10.1111/1467-6494.696169.

Van Raalte, J. L., Cunningham, J., Cornelius, A. E., \& Brewer, B. W. (2004). Environmental effects on social physique anxiety. Kinesiologica Slovenika, 10, 86-95.

Vormbrock, F., \& Neuser, J. (1983). Konstruktion zweier spezifischer Trait-Fragebogen zur Erfassung von Angst in sozialen Situationen (SANB und SVSS). [Construction of two specific trait questionnaires measuring social anxiety (SANB and SVSS).]

Diagnostica, 29, 165-182. 
Walling, M. D., Duda, J. L., \& Chi, L. (1993). The Perceived Motivational Climate in Sport Questionnaire : Construct and predictive validity. Journal of Sport \& Exercise Psychology, 15, 172-183.

Whiting, R. (1977). The chrysanthemum and the bat: Baseball samurai style. New York: Dodd.

Wylleman, P., \& Lavallee, D. (2004). A developmental perspective on transitions faced by athletes. In M. Weiss (Ed.), Developmental sport and exercise psychology: A lifespan perspective (pp. 507-527). Morgantown, WV: Fitness Information Technology.

Wylleman, P., \& Reints, A. (2010). A lifespan perspective on the career of talented and elite athletes: Perspectives on high-intensity sports. Scandinavian Journal of Medicine and Science in Sports, 20 (Suppl. 2), 88-94. doi: 10.1111/j.1600-0838.2010.01194.x.

Yoshida, T., Matsuo, T., Yamamoto, N., \& Taniguchi, Y. (1998). Japanese style of sports career development in athletes: Findings from cross-national research on athletes participating in the $18^{\text {th }}$ Universiade in 1995 at Fukuoka. Journal of Health Science, Kyushu University, 20, 63-76.

Zhang, J., Jensen, B. E. \& Mann, B. L. (1997). Modification and revision of the Leadership Scale for Sports. Journal of Sport Behavior, 20, 105-122. 
Table 1

Results of Univariate Analyses of Variance for Japanese-German Comparisons on Goal

Orientation, Fear of Negative Evaluation, and Perceived Coach Behavior

Variable

$F \quad \eta^{2}$

$M_{J}$

$S D_{J}$

$M_{G}$

$S D_{G}$

Task Orientation

$* 5.82$

0.02

3.23

0.46

3.43

0.41

Ego Orientation

$* * * 18.37$

0.12

3.11

0.57

2.64

0.63

Fear of Negative

2.74

0.02

2.92

0.88

2.67

0.36

Training and Instruction

$* * * 31.87$

0.19

3.37

0.74

4.05

0.56

Positive Feedback

$* * * 14.64$

0.10

3.59

0.71

4.05

0.65

Social Support

$* * * 20.62$

0.13

3.10

0.63

3.65

0.80

Democratic Behavior

0.11

0.00

3.37

0.71

3.41

0.76

Mastery Climate

0.17

0.00

4.01

0.60

3.97

0.54

Performance Climate

*** 16.91

0.11

3.06

0.76

2.50

0.77

Closeness

$* 4.05$

0.03

4.63

1.36

5.08

1.06

Satisfaction w.

Coach/Group

0.32

0.00

5.45

1.13

5.55

1.03

Notes. $d f=1,140 ; * p<.05 ; * * * p<.001 ; M_{\mathrm{J}}, M_{\mathrm{G}}$ and $S D_{\mathrm{J}}, S D_{\mathrm{G}}=$ means and standard deviations for the Japanese and German samples, respectively. 
Table 2

Intercorrelations between Perceived Coach Behaviors, Motivational Climate and Satisfaction as seen by Japanese and German Swimmers

\begin{tabular}{|c|c|c|c|c|c|c|c|c|}
\hline Coach Variables & (1) & (2) & (3) & (4) & (5) & (6) & (7) & (8) \\
\hline (1) Training and Instruction & & $.68 * *$ & $.74 * *$ & $.69 * *$ & $.60 * *$ & $.28 * *$ & $.51 * *$ & $.30 * *$ \\
\hline (2) Positive Feedback & $.60 * *$ & & $.68^{* *}$ & $.57 * *$ & $.55^{* *}$ & $.34 * *$ & $.64 * *$ & $.53 * *$ \\
\hline (3) Social Support & $.48 * *$ & $.47 * *$ & & $.71 * *$ & $.64 * *$ & $.22 *$ & $.49 * *$ & $.33 * *$ \\
\hline (4) Democratic Behavior & $.59 * *$ & $.50 * *$ & .29 & & $.62 * *$ & $.26 * *$ & $.40 * *$ & $.20^{*}$ \\
\hline (5) Mastery Climate & $.68 * *$ & $.46^{*}$ & $.42 *$ & $.62 * *$ & & $.29 * *$ & $.53^{* *}$ & $.35^{* *}$ \\
\hline (6) Performance Climate & $-.33^{*}$ & -.04 & $-.38 *$ & -.08 & $-.36 * *$ & & $.33 * *$ & .17 \\
\hline (7) Closeness & $.56^{* *}$ & $.43 * *$ & $.74 * *$ & $.50 * *$ & $.51 * *$ & $-.27 *$ & & $.606^{* *}$ \\
\hline (8) Athletes' Satisfaction & .06 & .16 & .02 & -.03 & .08 & -.02 & .21 & \\
\hline
\end{tabular}

Notes. Correlations above the diagonal correspond to the Japanese sample; correlations below the diagonal correspond to the German sample

$* p<.05 ; * *<.01$ 